\title{
Characterization of the steady and unsteady spray structures of a liquid jet in supersonic crossflow
}

\author{
Dayna Obenauf*1, James Braun ${ }^{1}$, Terrence R. Meyer ${ }^{1}$, Guillermo Paniagua ${ }^{1}$, Paul E. Sojka ${ }^{1}$, \\ and Francois Falempin ${ }^{2}$ \\ ${ }^{1}$ School of Mechanical Engineering, Maurice J. Zucrow Laboratories, Purdue University, \\ West Lafayette, IN, USA \\ ${ }^{2}$ MBDA, Le Plessis Robinson, France
}

\begin{abstract}
Characterization of the spray resulting from a $1 \mathrm{~mm}$ diameter liquid ethanol jet injection into a supersonic air crossflow is presented. The isentropic Mach number at the injection location is 1.94 , while the momentum flux ratio is varied between 3 and 13. Steady and unsteady components of the penetration depth are retrieved using backlit imaging perpendicular to the spray at a frame rate of $20 \mathrm{kHz}$, coupled with a telecentric lens to accommodate the test section's width and ensure equal magnification through the spray's width. Spray width at specific axial locations downstream of injection is measured by imaging Mie scattered light from a planar laser sheet directed perpendicular to the flow direction so that a cross section of the spray is recorded by a camera placed at a known angle between camera axis and flow direction.
\end{abstract}

Keywords: Jet breakup, supersonic crossflow

\section{Introduction}

The breakup of a liquid jet in a supersonic crossflow has been of interest to many researchers for over 50 years. $[1,2,3]$ Understanding the liquid atomization process is essential for predicting the appropriate time and length scales of the primary and secondary atomization processes to better model fuel and air mixing in combustion processes. As a result, numerous groups have attempted experimental measurements and computation modelling of the liquid jet breakup process when injected into a supersonic crossflow. However, this fundamental multiphase process continues to be challenging to thoroughly understand due to the difficulty of capturing complex physics of dense particle fields with length scales on the order of microns and timescales in nanoseconds.

Preliminary studies began with experimental measurements to predict spray penetration depth via backlit and shadowgraph techniques [1,3] and small injector diameter tests allowed for size predictions of shedding droplets off the expanding jet. [2] Sauter mean diameter (SMD) of the droplets on the edge of the plume were also measured using holography and shadowgraphy [4], and phase Doppler particle analysis (PDPA) [5], with discrepancies greater than $300 \%$ in results between the studies. In all these studies, liquid properties, injector angle, and aeration of the liquid prior to injection were all evaluated to present a variety of breakup conditions. However, many more variable that have potential to affect jet breakup have yet to be evaluated, including test section static pressure, multiple injector sizes to compare the effect of liquid mass flow rate, and measurement of the spray at large distances downstream of injection.

This present study attempts to fill in some of these gaps by implementing a variety of measurement techniques to both qualitatively and quantitatively describe the spray and surrounding air flow. Air flow rates and liquid mass flow rates are varied to generate a wide range of momentum flux ratios and static pressures. Highspeed backlit imaging and shadowgraphy are used to observe the spray interactions with the surrounding air flow and measure spray depth. Recording of Mie scattered light at select locations downstream of injection allows for measurement of spray width, a characteristic that has yet to be described in previous studies.

\section{Experimental apparatus}

All measurements were performed in the Linear Experimental Aerothermal Facility (LEAF) of the Purdue Experimental Turbine Aerothermal Lab (PETAL). The facility can provide air mass flows up to $30 \mathrm{~kg} / \mathrm{s}$, air temperatures up to $700 \mathrm{~K}$ and pressures up to 8 bar in the settling chamber. The high-pressure line provides air from a $56 \mathrm{~m}^{3}$ air storage, stored at 137 bar [6]. Downstream, a vacuum tank (volume $283 \mathrm{~m}^{3}$ ) can deliver vacuum levels down to 6 mbar. The LEAF facility, with a cross section of 0.17 by 0.23 is depicted in the Figure below. Quartz windows can be installed on either side of the test section allow for full optical access for all diagnostics of interest to characterize the spray. To deliver supersonic flows, a converging-diverging nozzle, with a diverging angle of 7.38 degrees was manufactured, as shown in the Figure below. The throat length is $0.0254 \mathrm{~m}$, measured with an inside dial caliper with a precision of $+-0.013 \mathrm{~mm}$. The converging-diverging nozzle is mounted from the bottom of the LEAF wind tunnel. The Mach number throughout the accelerating passage is highlighted in the below Figure. Mach number at the aft the nozzle reaches 2.5. The injection of liquid sprays is provided through the top plate of the test section at a location with a flow speed of $M_{\text {isentropic }}=1.94$. 
a)
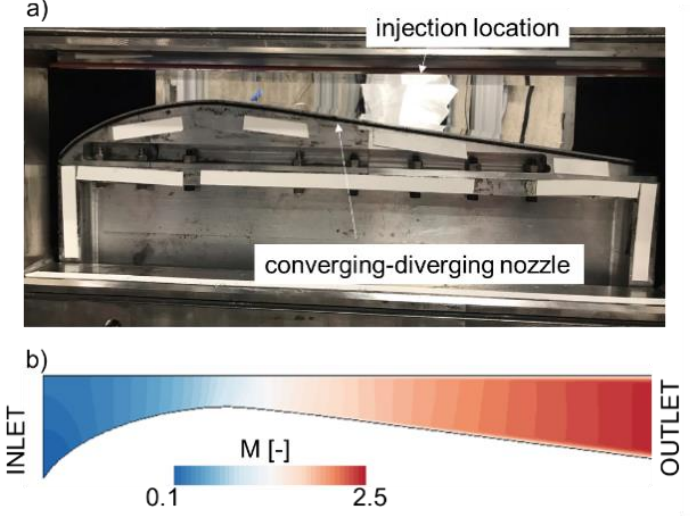

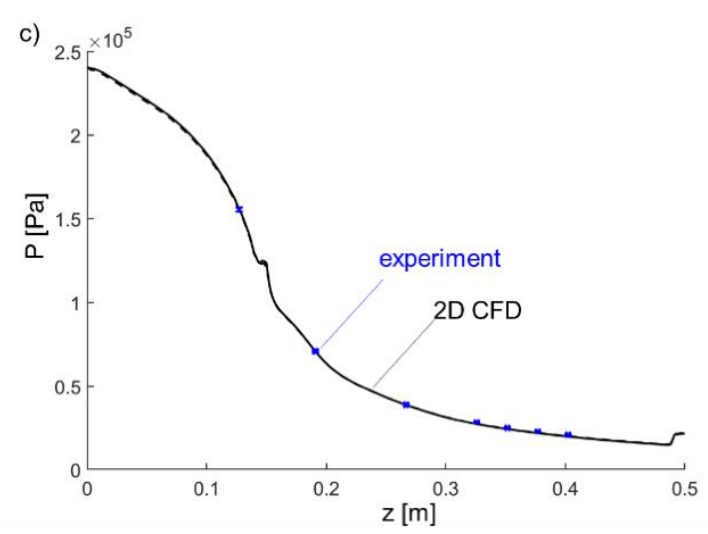

Figure 2. a) Converging-diverging nozzle, b) Mach number from 2D Reynolds Averaged Navier Stokes simulations, c) pressure trace from CFD and experiment.

The converging-diverging nozzle was equipped with static pressure taps, to measure the static pressure along several axial and tangential spans. Scanivalve DSA pressure scanners were used, with an accuracy of +$200 \mathrm{~Pa}$. Sixteen pressure measurements were recorded at several axial and tangential locations. Error between CFD and experiment had a maximum discrepancy below $5 \%$. At one constant axial location and across $90 \%$ span of the test section, variability of pressure was below $5 \%$. The discrepancy of isentropic Mach number at the injection point was below $2 \%$ (Figure 2 c).

Liquid ethanol (100\%) was stored into a high-pressure aluminum SCUBA tank with an internal volume of $12.9 \mathrm{~L}$. The tank was pressurized using facility nitrogen supplied at 414 bar and regulated using a TESCOM ER5000 Series Electropneumatic Actuator to the necessary pressure to achieve desired flow rate. Typical tank pressures ranged from approximately 5.5-10.3 bar. A dip tube ran ethanol from the bottom of the tank to a pneumatically actuated solenoid valve, used to start and stop injection into the test section. A Druck UNIK5000 pressure transducer and K-type thermocouple were placed downstream of the valve to measure ethanol pressure and temperature before flowing into a cavitating venturi. Desired mass flow rates were targeted by calculating the necessary upstream pressure at the inlet of the venturi. The pressures were achieved by calibrating the necessary control regulator set pressure to accommodate for minor pressure losses. From the venturi, the ethanol flowed directly into the injector, which consisted of one single injection hole with a $1 \mathrm{~mm}$ diameter and a length-to-diameter ratio of 12. A plenum at the start of the injection tube helped to reduce the potential for fluid cavitation within the injector.

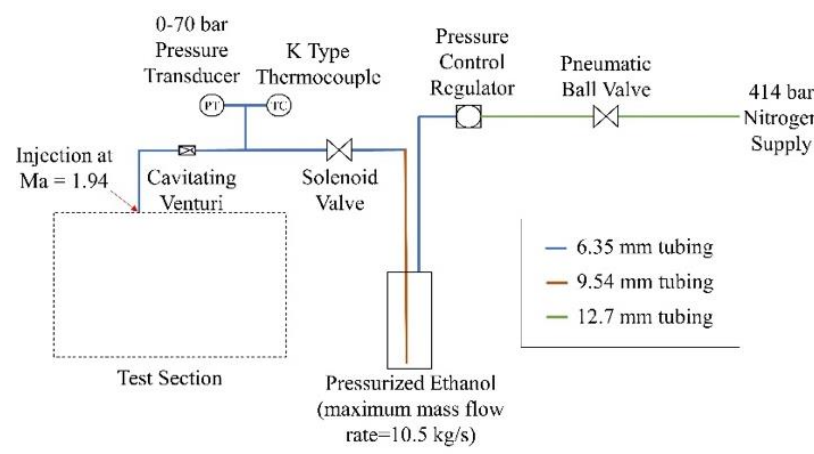

Figure 3. Pressurized tank and instrumentation for injection liquid ethanol into the test section.

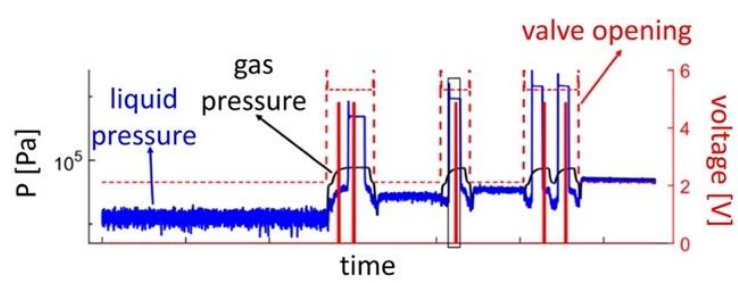

Figure 4. Syncing of the data (liquid pressures, gas pressures, valve opening, and camera trigger.

Figure 4 shows the syncing of all relevant data. Liquid pressures, gas pressures within the test section, the opening of the valve as well as the recording of the camera were recorded as a function of time. Pressure and temperature data were acquired at $200 \mathrm{~Hz}$. The liquid temperature was around 290K, the gas temperature was around $290 \mathrm{~K}$ at the onset of the experiment and decreased due to expansion within the high-pressure storage tank.

\section{Backlit Imaging}


A 50-W diffuse LED was placed on one side of the test section, with the Photron SA-Z Fastcam on the other side, such that images were captured perpendicular to the flow direction. All images were recorded at $20 \mathrm{kHz}$ at a full resolution of $1024 \times 1024$ pixels. Due to the width of the spray and the test section, a TitanTL Telecentric Lens with primary magnification of $0.238 \mathrm{X}$, a working distance of $267 \mathrm{~mm}$, and a depth of field of $\pm 14.9 \mathrm{~mm}$ was used to maintain equal magnification of the spray. Pixel size was determined using a dot grid with 1-mm spacing, placed equally spaced between optical windows to be at the center of the telecentric lens in-focus range. Pixel size was $0.0842 \mathrm{~mm}$.

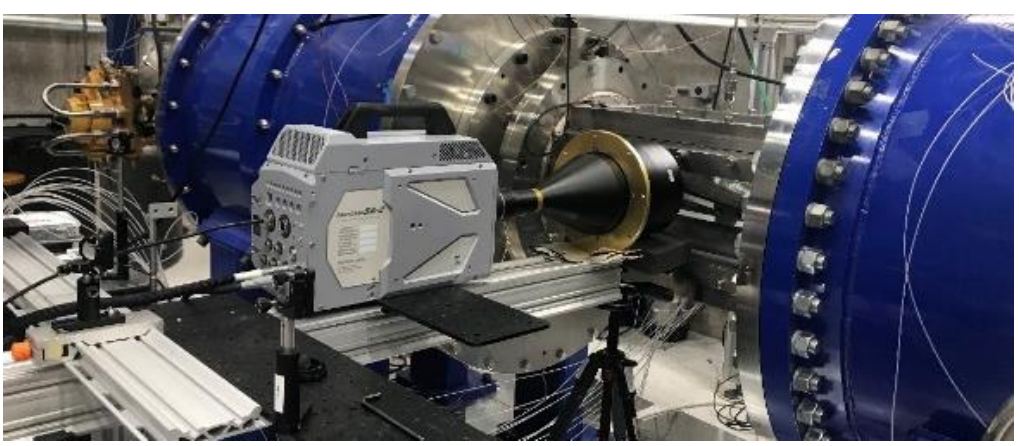

Figure 5. Telecentric lens used for maintaining constant magnification across the width of the spray.

The figure below depicts the procedure to extract the spray depth as a function of axial distance. Background pictures were taken for each test and the raw image with spray is then subtracted to obtain the background subtracted figure. Through binarization, the spray depth is calculated at each axial location. On average, 836 images were required to reach convergence.

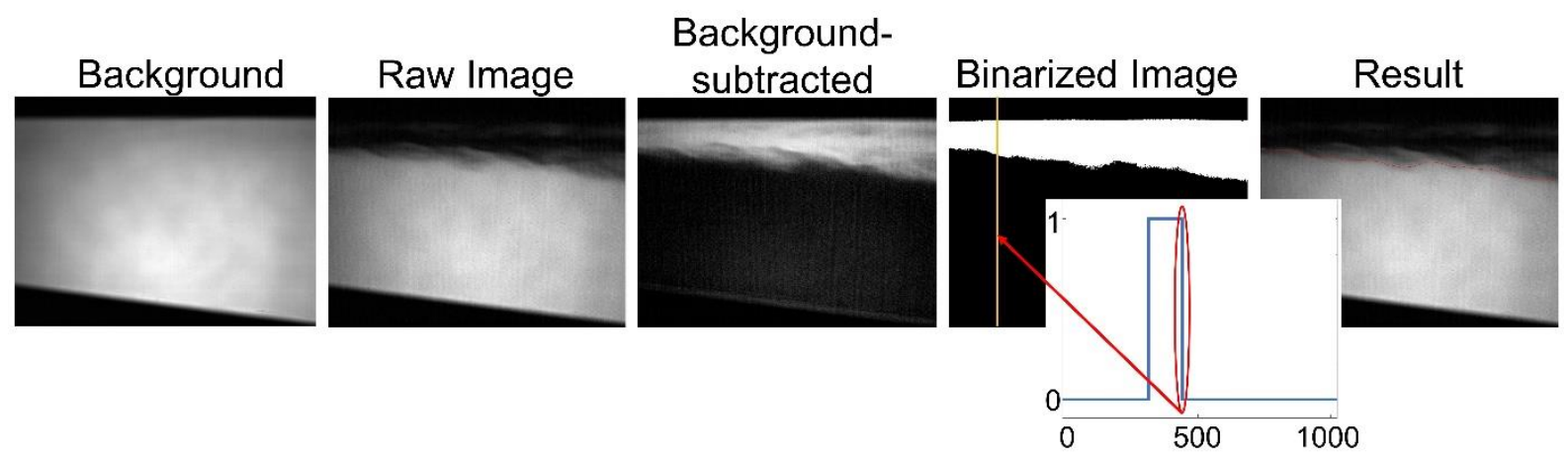

Figure 6. Post-processing routine to extract the unsteady spray depth at each axial location.

\section{Shadowgraphy}

The light from a diffuse broad spectra white LED was focused using two plano-convex lenses, the first being a $25.4-\mathrm{mm}$ diameter lens of $f=150 \mathrm{~mm}$ and the second being a $100-\mathrm{mm}$ diameter lens of $f=100 \mathrm{~mm}$. A pinhole was placed at the point where the light was focused to create a point source. After expansion from the pinhole, a 100$\mathrm{mm}$ diameter lens with a focal length of $f=400 \mathrm{~mm}$ was used to collimate the light before entering the test section perpendicular to the flow direction. The TitanTL Telecentric Lens and Photron SA-Z Fastcam were placed on the opposite side of the test section to capture the. From the shadowgraphs, shock wave angles and instabilities of the bow shock forming in front of the shock and lambda shocks on the instability waves on the front of the spray could be measured.

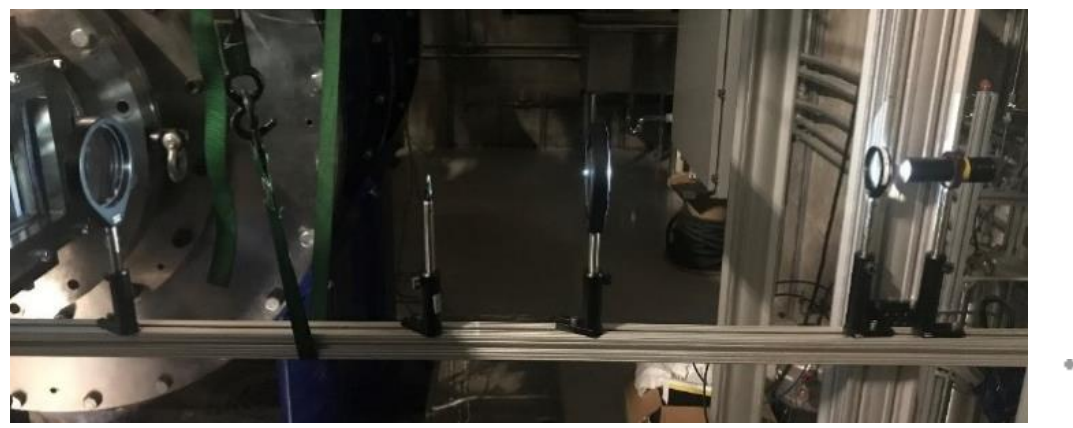


Figure 7. Lens system used to collimate a 100-mm diameter beam from the diffuse LED source.

\section{Mie Scattering}

A planar laser sheet was directed perpendicular to the spray at known downstream locations of injection to image the light scattering off the ethanol droplets. A $10 \mathrm{~Hz}$ laser at $9 \mathrm{~mJ} /$ pulse was focused using a plan-convex lens of $f=1000 \mathrm{~mm}$ at the center of the test section, and expanded in one dimension using a cylindrical lens of $f=175$ $\mathrm{mm}$. An Andor Zyla $5.5 \mathrm{sCMOS}$ camera with 5-megapixel resolution was placed such that it formed an angle of 25 degrees with the laser sheet. Images were perspective oriented to show the width and height of the spray.
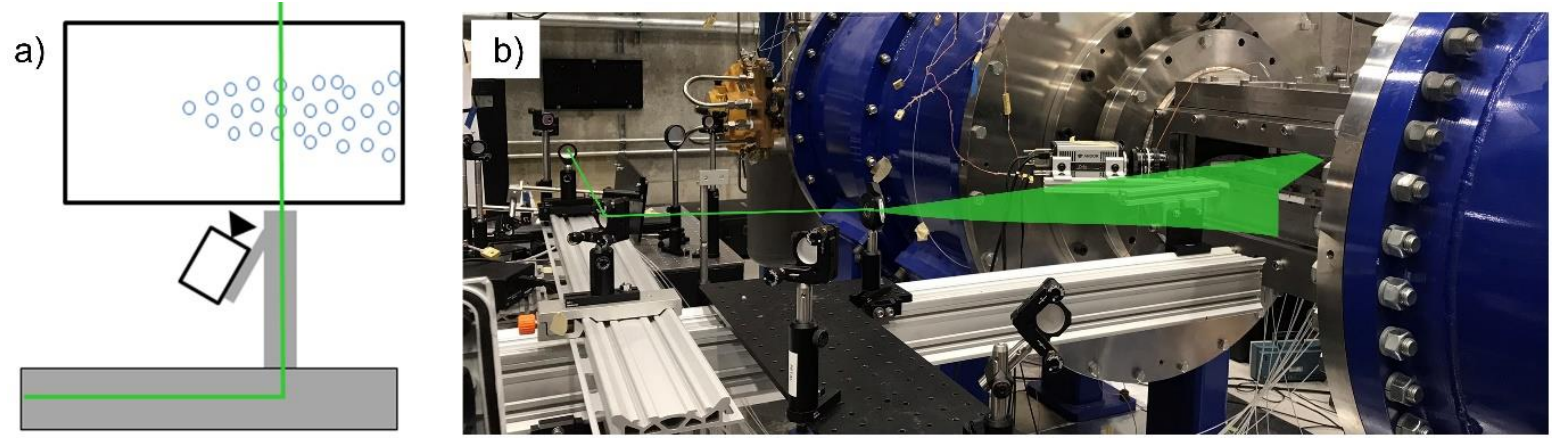

Figure 8. a) Illustration of the camera angle relative to the laser sheet, b) laser path.

The calibration procedure for the Mie Scattering is shown in the figure below through Davis by Lavision software. A calibrated dot pattern (dot distance of $5 \mathrm{~mm}$ and a dot diameter of $1.2 \mathrm{~mm}$ ) was used to De-warp the image from raw to real world using a known scale from a dot board.
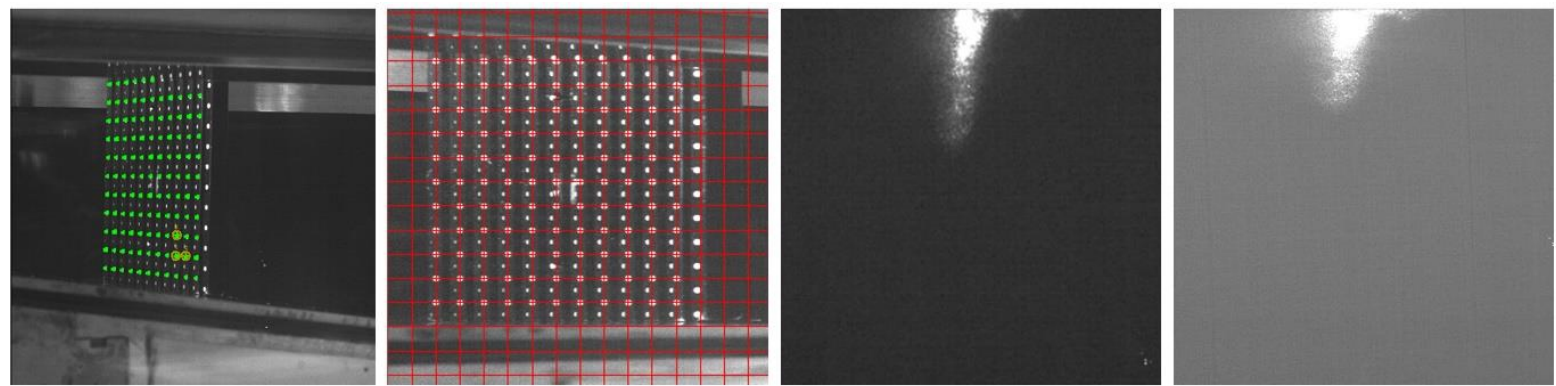

Figure 9. Calibration procedure for the Mie scattering perspective re-orientation.

An example to retrieve the spray width and depth from the Mie Scattering is shown below in Figure 10. First, predicted spray depth is used to determine the grayscale threshold that should be used for each case to determine the edges of the spray. Then, each row of pixels is evaluated to determine the number of pixels that fall between those that meet the grayscale threshold criterion. The graph shows how this width varies with vertical distance from the injection plate. The lines indicate the average, minimum, and maximum spray depth from backlit imaging results under similar conditions.
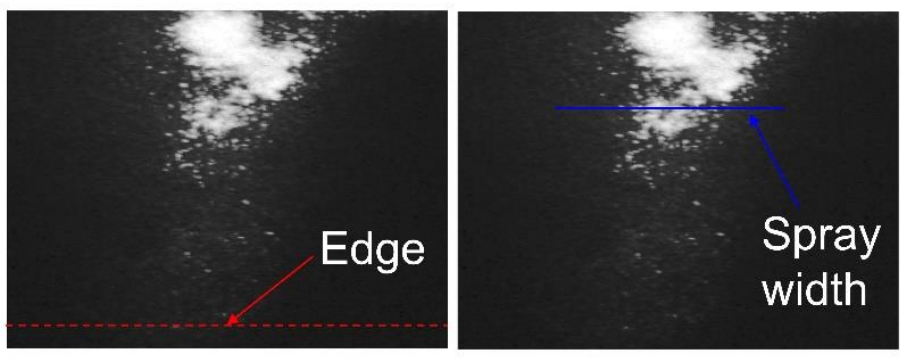

Figure 10. Postprocessing method to extract average width and depth of the spray.

\section{Spray width $(\mathrm{mm})$}

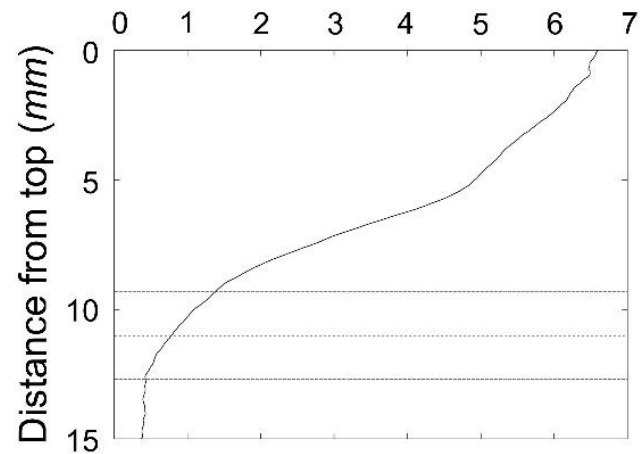

\section{Results and Discussion}


Using backlit imaging with the telecentric lens, 17 separate cases were tested. Eleven tests were set to image a location $10 \mathrm{~cm}$ downstream of injection, and the remaining six were set at $15 \mathrm{~cm}$ downstream of injection. Average momentum flux ratios ranged from 3.95 to 13.17 . Static pressures ranged from 0.319 bar to 0.434 bar, where ten of the tests targeted a lower mass flow rate of air $(\sim 0.3 \mathrm{~kg} / \mathrm{s})$ and the remaining targeted higher air mass flow rates $(\sim 0.4 \mathrm{~kg} / \mathrm{s})$. Figure 11 below shows the results, comparing the spray penetration depth in $\mathrm{mm}$ vs the momentum flux ratio. The graph on the left displays the spray depth at $10 \mathrm{~cm}$ downstream of injection, and the graph on the right at $15 \mathrm{~cm}$. The horizontal bars represent the minimum to maximum range of momentum flux ratios over the 0.1 second duration of imaging, and the vertical bars show two standard deviations of the spray depth.

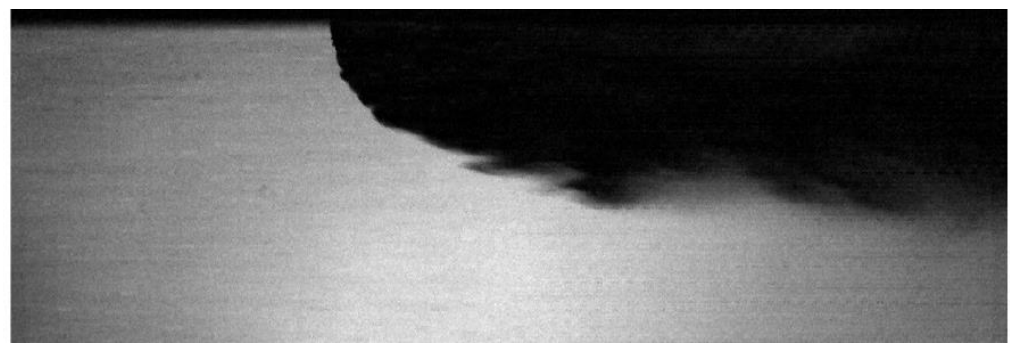

Figure 11. Backlit imaging of the spray at injection.

In both cases, the results showed a strong trend of spray depth increasing linearly with momentum flux ratio. In Figure 12, a linear fit was added to both graphs, and the slope of this line decreased with downstream distance from the point of injection, indicating that spray depth is much more dependent on momentum flux ratio closer to injection. The results showed little dependence on test section static pressure.

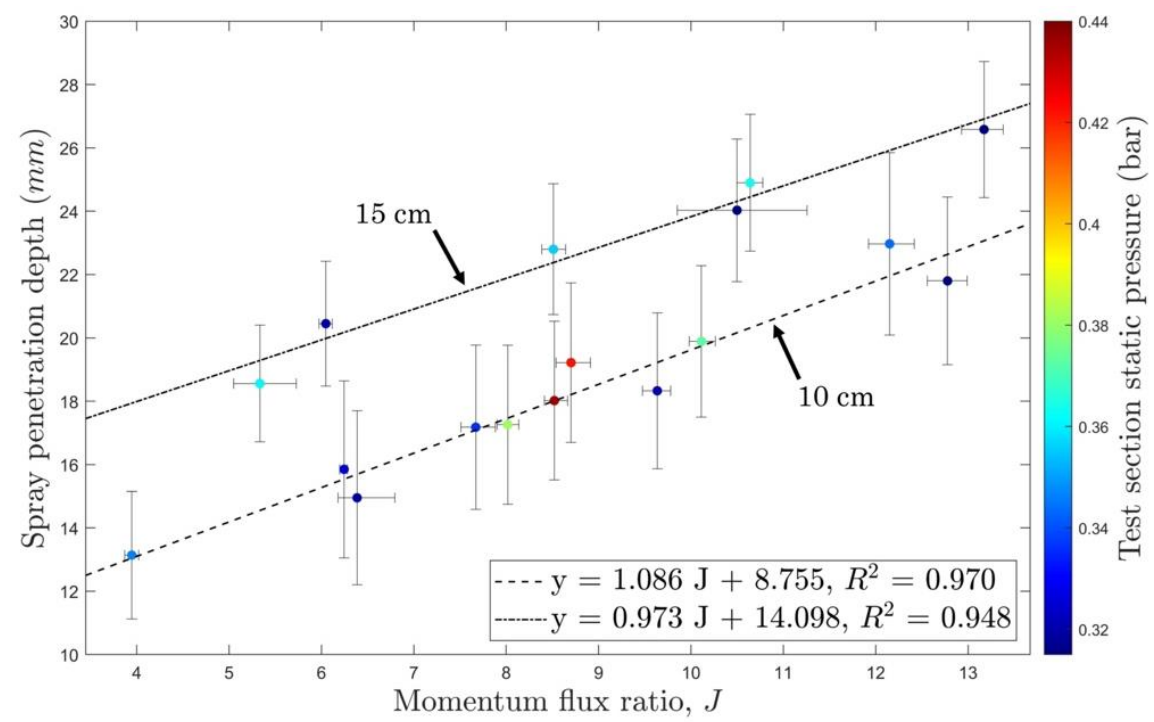

Figure 12. Spray penetration depths vs. momentum flux ratios, measured from backlit imaging at 10 and $15 \mathrm{~cm}$ downstream of injection.

Figure 13 shows the results of how spray penetration depth varies with downstream distance, where the vertical bars on each point represent the range of two standard deviations of the spray depth from all 2,000 images. Between 60 and $130 \mathrm{~mm}$, the depth tends to increase with distance, and the depth tends to become slightly steadier after $140 \mathrm{~mm}$. 


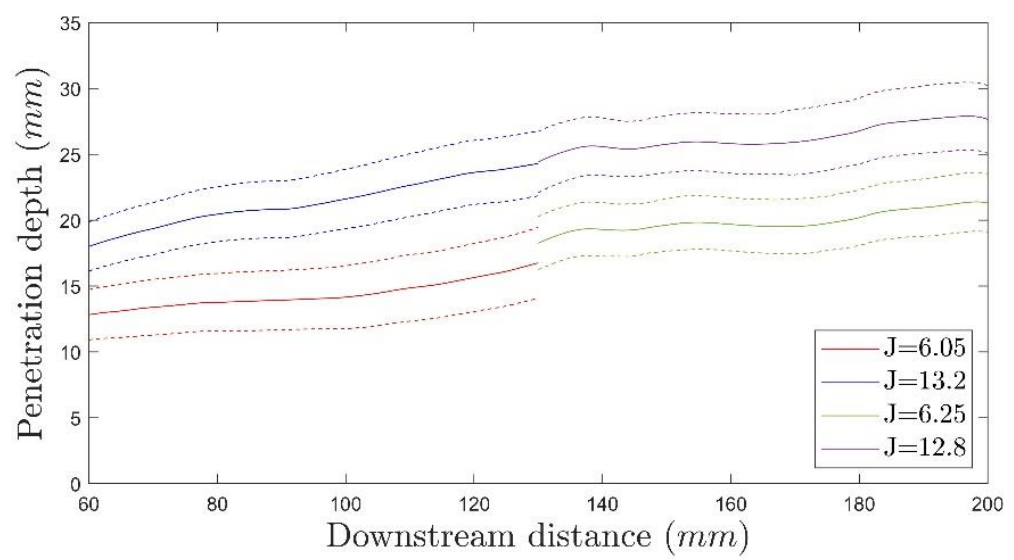

Figure 13. Spray penetration depths vs. downstream distance, measured from backlit imaging at four different momentum flux ratios.

The linear fit equation was used to predict spray depth at the average momentum flux ratio for each case where Mie scattered light was measured from the laser sheet perpendicular to the flow direction. The results were used to measure the average grayscale for each case that was then used to determine the horizontal edges of the spray to predict spray width. After running this routine, the maximum depth at which a width could be predicted was plotted, shown below in Figure 14. The same linear fit that was calculated from the backlit imaging results in Figure 11 was plotted on the Mie scattering results graph, demonstrating good agreement the two measurement techniques. Additionally, this agreement supports the accuracy of the spray width results. Figure 15 shows examples of the Mie Scattered light images, where the first image shows a time-averaged image of all images collected for one test, and the next five images show the results at individual times, with 0.1 seconds between frames.

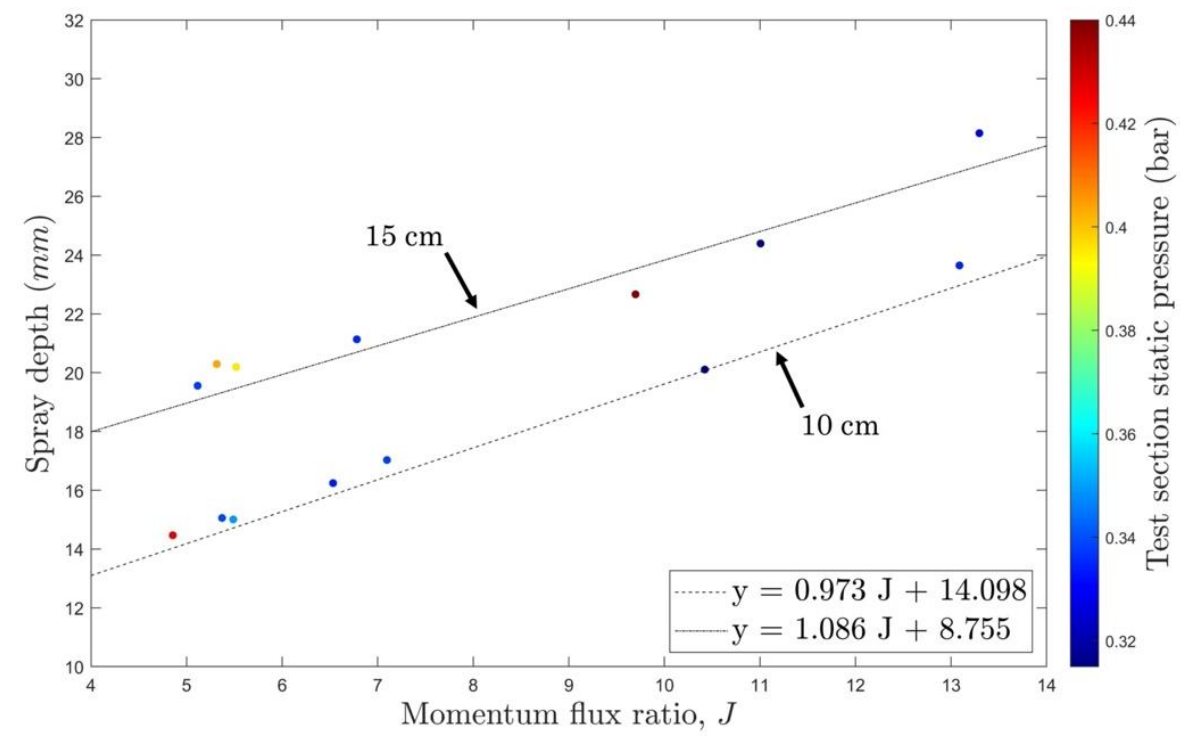

Figure 14. Spray penetration depths vs. momentum flux ratios, measured from Mie Scattering at 10 and $15 \mathrm{~cm}$ downstream of injection. Note the equation for the line is from the backlit imaging linear fit in Figure 11. 

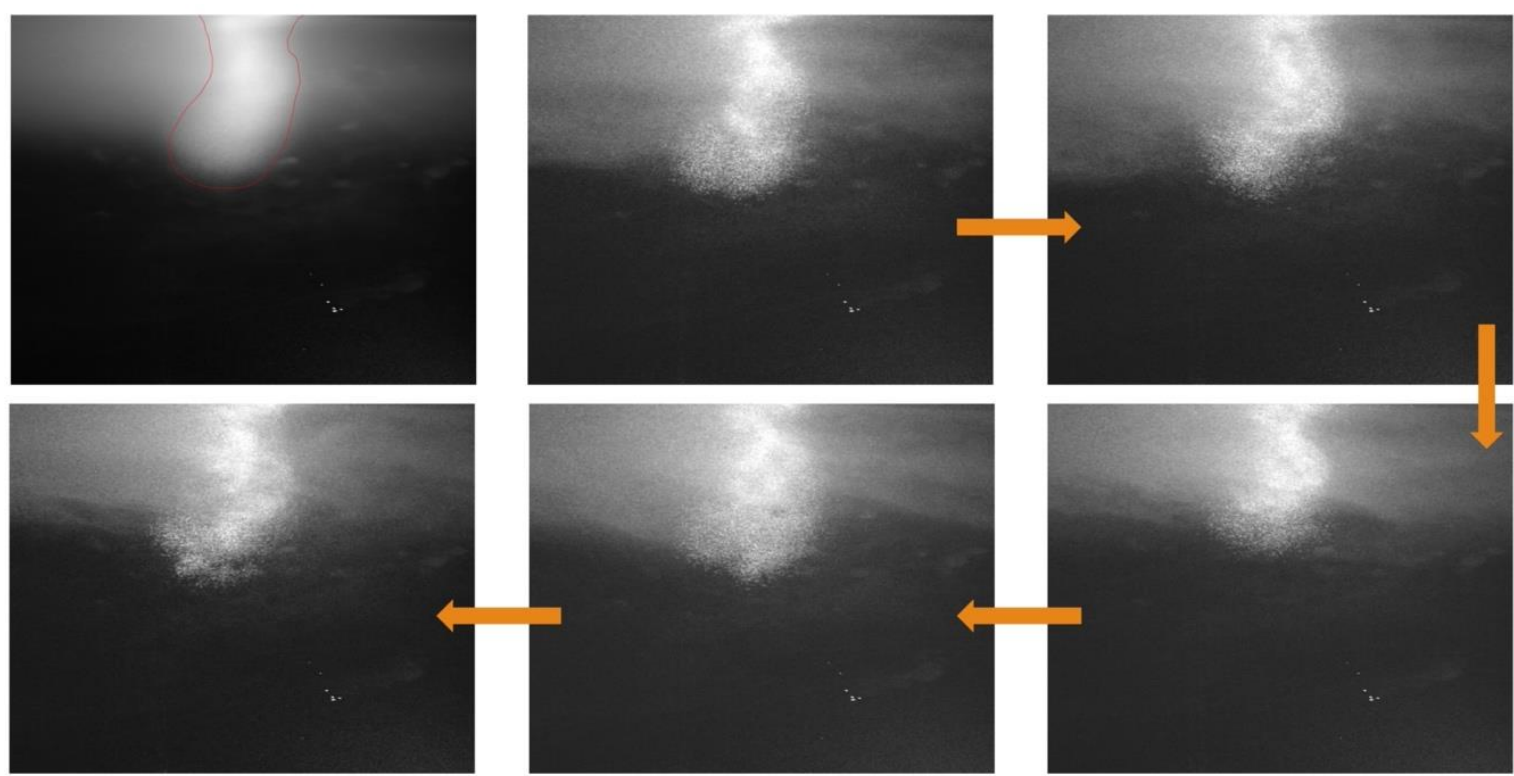

Figure 15. Resulting Mie scattering images, where the top-left image shows the average of all 50 images collected for one test, and the spray edge identified by the red line. The remaining images show a sequence of five consecutive frames to demonstrate the unsteady features of the spray.

The spray width results are shown below in Figure 16. Seven cases were recorded at $10 \mathrm{~cm}$ downstream of injection, and seven recordings at $15 \mathrm{~cm}$. Five cases were recorded with high air mass flow rates, and therefore higher static pressures in the test section. Overall, spray width was found to increase both with momentum flux ratio and with downstream distance from the injection point.

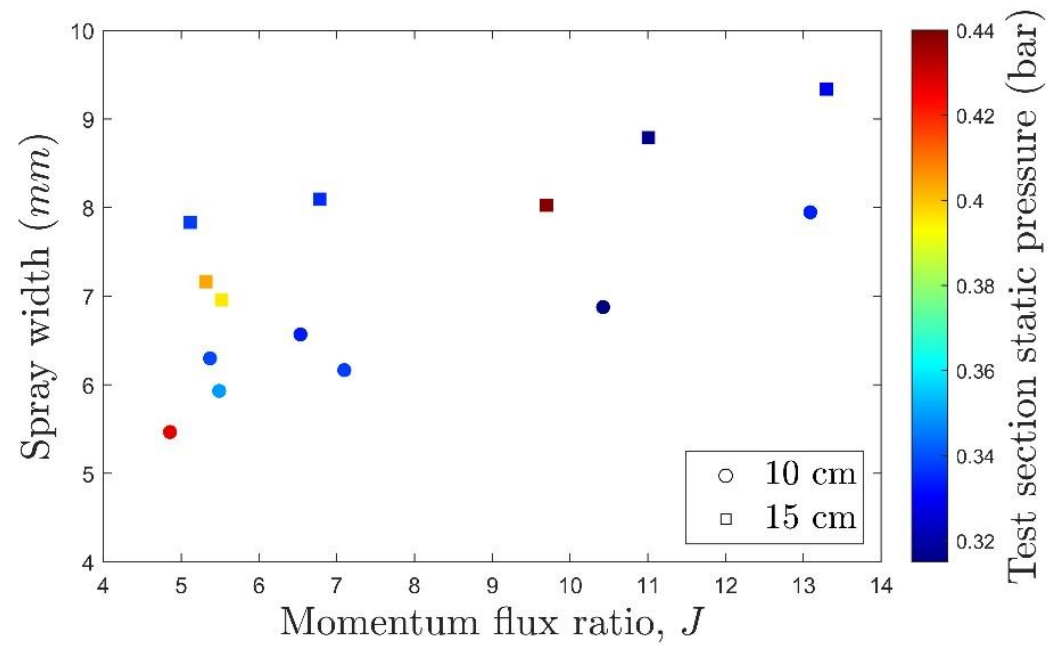

Figure 16. Spray penetration widths vs. momentum flux ratios, measured from Mie Scattering at 10 and $15 \mathrm{~cm}$ downstream of injection.

Shadowgraph images were recorded at the point of injection to evaluate the boundary layer in this region and observe the spray interactions with the surrounding flow. An unsteady bow shock was formed in front of the spray, with small legs to the concave parts of the instability waves that formed on the edge of the spray plume, particularly in the region closest to the injection point. 


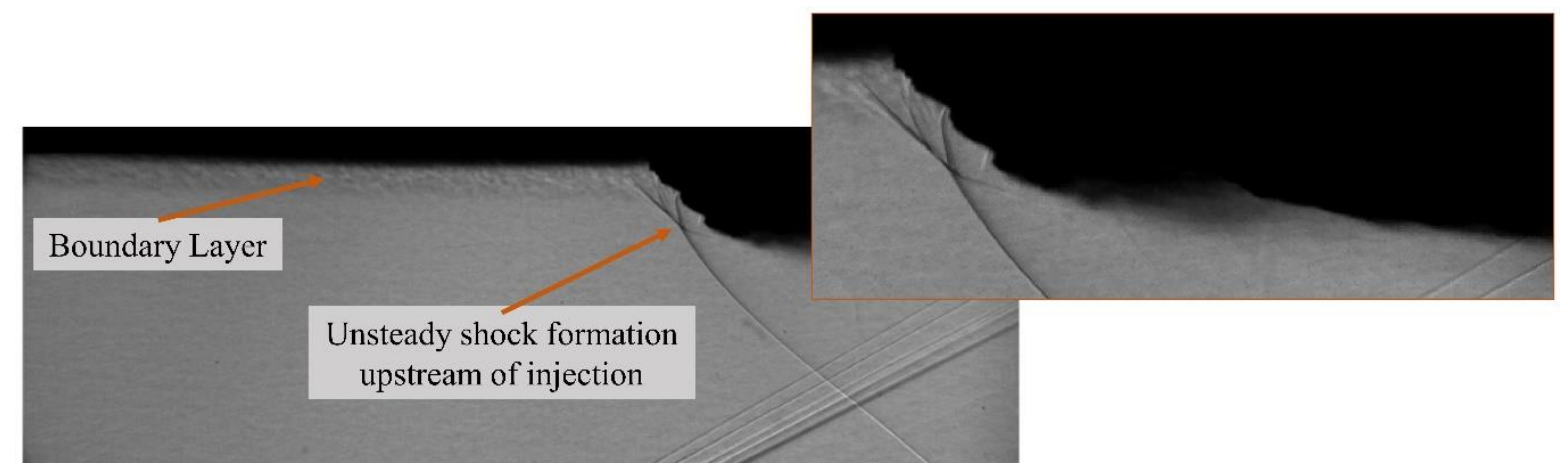

Figure 17. Shadowgraph image taken at the point of injection.

\title{
Conclusions
}

Liquid ethanol was injected into a supersonic crossflow through a $1 \mathrm{~mm}$ diameter hole in the top plate of the test section. The Mach number at the point of injection was 1.94, and air and liquid mass flow rates were varied to produce momentum flux ratios between 3 and 13, with static pressures in the test section ranging from 0.32 to 0.44 bar. The resulting spray was measured using a variety of measurement techniques. The first was a backlit imaging approach using a high-speed camera to record at $20 \mathrm{kHz}$, using a telecentric lens that maintained constant magnification over a range of distances from the camera that contained the spray. These results were presented to show the average spray depth and variation over 2,000 images collected. It was found that spray depth increased linearly with momentum flux ratio, and the slope of this linear regression decreased with downstream distance from injection.

Spray width, a characteristic that has not previously been discussed in other studies, was measured by imaging the Mie scattered light off the droplets from a planar laser sheet directed into the test section perpendicular to liquid and air flow. The camera was placed at a known angle relative to the axial direction of flow, and the images were perspective re-oriented using specialized software. Spray depths and widths at these specific downstream locations were calculated by selecting a grayscale threshold that was determine from the backlit imaging results. The resulting spray depths measured from backlit imaging and Mie scattering were in good agreement. Spray widths were found to increase both with momentum flux ratio and distance downstream from injection.

Lastly, shadowgraphy was used to observe the surrounding air flow and interactions between the liquid jet and air at the point of injection. Prominent bow shocks could be observed in all cases. Further analysis is needed to better understand how these high-frequency flow instabilities vary and affect the spray instability waves that promote breakup.

\section{Acknowledgments}

We thank Matthew Hoeper for providing tools and knowledge for implementing the Mie scattering measurement technique.

\author{
Nomenclature \\ M Mach Number \\ $J \quad$ Momentum Flux Ratio
}

\section{References}

[1] Forde, J. M., S. Molder, and E. J. Szpiro. "Secondary liquid injection into a supersonic airstream." Journal of Spacecraft and Rockets 3.8 (1966): 1172-1176.

[2] Sherman, Allan, and Joseph Schetz. "Breakup of liquid sheets and jets in a supersonic gas stream." AIAA Journal 9.4 (1971): 666-673.

[3] Kush Jr, Edward A., and Joseph A. Schetz. Decomposition of a Liquid Jet Injected Normal to a Supersonic Air Stream. VIRGINIA POLYTECHNIC INST AND STATE UNIV BLACKSBURG DEPT OF AEROSPACE ENGINEERING, 1972.

[4] Sallam, K. A., Christian Aalburg, and G. M. Faeth. "Breakup of round nonturbulent liquid jets in gaseous crossflow." AIAA journal 42.12 (2004): 2529-2540.

[5] Lin, Kuo-Cheng, et al. "Structures and temporal evolution of liquid jets in supersonic crossflow." 55th AIAA Aerospace Sciences Meeting. 2017.

[6] Paniagua, G., et al. "Design of the Purdue Experimental Turbine Aerothermal Laboratory for Optical and Surface Aerothermal Measurements." Journal of Engineering for Gas Turbines and Power 141.1 (2019). 